\title{
Survival analysis of a stochastic delay single-species system in polluted environment with psychological effect and pulse toxicant input
}

\author{
Xiangjun Dai ${ }^{*}$, Suli Wang ${ }^{2}$, Weizhi Xiong ${ }^{1}$ and $\mathrm{Ni} \mathrm{Li}^{1}$
}

"Correspondence:

daiaga0921@126.com

'School of Data Science of TongRen

University, 554300 Tongren,

P.R. China

Full list of author information is

available at the end of the article

\section{Springer}

\begin{abstract}
We propose and study a stochastic delay single-species population system in polluted environment with psychological effect and pulse toxicant input. We establish sufficient conditions for the extinction, nonpersistence in the mean, weak persistence, and strong persistence of the single-species population and obtain the threshold value between extinction and weak persistence. Finally, we confirm the efficiency of the main results by numerical simulations.
\end{abstract}

Keywords: Psychological effect; Persistence; Extinction; Polluted environment

\section{Introduction}

Along with fast development of agriculture and modern industry, a large number of toxic pollutants are discharged into the ecosystem, and therefore it is an undeniable fact that environmental pollution becomes increasingly serious, such as pollution of pollutants from burning agricultural plant straw, heavy metal pollution, water pollution caused by crop fertilization and pesticide application. As it is well known, the existence of various poisons are becoming a threat to the survival of unprotected populations, which has prompted many scholars to investigate the impact of toxins on the population and assess the risk of the population. An important tool to analyze the effects of toxins on population is establishing a mathematical model [1-7].

Wei and Chen [8] proposed a mathematical model for the first time to study the physiological effects of vertebrates on population in a polluted environment. The so-called "psychological" effect refers to: in the heavy pollution environment, because the organism with spine has a good sensory nervous system, which can transmit the information of the polluted environment to the area where the brain can explain the information, the effective contact between the organism and the live environment will be reduced, which plays the role of self-protection; for instance, fish can identify the information in the polluted environment through their own neurosensory system and make decisions to either escape from the polluted area or bear the environmental toxicant [8]. Afterwards, Lan and Wei

(c) The Author(s) 2020. This article is licensed under a Creative Commons Attribution 4.0 International License, which permits use sharing, adaptation, distribution and reproduction in any medium or format, as long as you give appropriate credit to the original author(s) and the source, provide a link to the Creative Commons licence, and indicate if changes were made. The images or other third party material in this article are included in the article's Creative Commons licence, unless indicated otherwise in a credit line to the material. If material is not included in the article's Creative Commons licence and your intended use is not permitted by statutory regulation or exceeds the permitted use, you will need to obtain permission directly from the copyright holder. To view a copy of this licence, visit http://creativecommons.org/licenses/by/4.0/. 
[9] considered that the exogenous input of toxins is regular in some practical situations. They proposed the following single-species population model with psychological effect and impulsive toxicant in a pollution environment:

$$
\left\{\begin{array}{l}
\dot{x}(t)=x(t)\left(r-r_{0} c_{0}(t)-a x(t)-\frac{\lambda c_{e}(t)}{1+\alpha c_{e}^{2}(t)}\right), \\
\dot{c}_{0}(t)=\left(k c_{e}(t)-(g+m+b) c_{0}(t)\right), \\
\dot{c}_{e}(t)=-h c_{e}(t), \quad t \neq n \gamma, n \in z^{+}, \\
\Delta x(t)=0, \quad \Delta c_{0}(t)=0, \quad \Delta c_{e}(t)=\mu, \quad t=n \gamma, n \in z^{+},
\end{array}\right.
$$

where $x(t)$ represents the density of the population, $c_{0}(t)$ and $c_{e}(t)$ represent the concentration of toxins in the organism and the concentration of toxins in the environment, respectively, $r$ and $r_{0}$ stand for the net growth rate in nonpolluted environment and response intensity of biological growth to toxins, $k c_{e}(t)$ stands for the uptake of toxins in the environment, $g c_{0}(t)$ represents the emission rate of toxins, $m c_{0}(t)$ represents the purification rate of toxins because of metabolic process of organisms, $b c_{0}(t)$ is the loss due to giving birth at time $t,-h c_{e}(t)$ denotes the amount of reduction in the purification of toxins by the environment itself, $\mu$ and $\gamma$ denote the toxicant input amount and the period of pulse input toxin, respectively, and $\Delta \phi(t)=\phi\left(t^{+}\right)-\phi(t)$.

In reality, population growth is more or less disturbed by environmental factors, such as temperature, humidity, and seasonal climate change, and almost all the observed data show that there are obvious random fluctuations in the growth process of organisms. Therefore, in some practical cases, ignoring the randomness of the system and using deterministic models to describe and predict the system behavior are not always satisfactory; especially, it is not suitable to use deterministic population model to study how to protect endangered species [10]. May [11] pointed out that due to the impact of environmental noises, the birth rate, death rate, carrying capacity, competition coefficients, and other parameters involved with the system exhibit random fluctuation to a greater or lesser extent [12-17]. On the other hand, the influence of delay has played an important role in the population dynamic [18-22].

In this paper, we suppose that the environmental noises affect all parameters of model (1) (see e.g. $[23,24]$ ). Considering the work of population psychological effect, we propose the following stochastic impulsive single-species population model with delay and psychological effect in polluted environment:

$$
\left\{\begin{aligned}
d x(t)= & x(t)\left(r-r_{0} c_{0}(t)-a x(t)-c x(t-\tau)-\frac{\lambda c_{e}(t)}{1+\alpha c_{e}^{2}(t)}\right) d t \\
& +\sigma_{1} x(t) d B_{1}(t)+\sigma_{2} c_{0}(t) x(t) d B_{2}(t)+\sigma_{3} x^{2}(t) d B_{3}(t) \\
& +\sigma_{4} x(t) x(t-\tau) d B_{4}(t)+\frac{\sigma_{5} x(t) c_{e}(t)}{1+\alpha c_{e}^{2}(t)} d B_{5}(t), \\
d c_{0}(t)= & \left(k c_{e}(t)-(g+m+b) c_{0}(t)\right) d t, \\
d c_{e}(t)= & -h c_{e}(t) d t, \quad t \neq n \gamma, n \in z^{+}, \\
\Delta x(t)= & 0, \quad \Delta c_{0}(t)=0, \quad \Delta c_{e}(t)=\mu, \quad t=n \gamma, n \in z^{+},
\end{aligned}\right.
$$

where $\tau$ represents the time delay, $B_{i}(t)$ stands for a standard Brownian motion defined on a complete probability space $(\Omega, F, P)$ with filtration $\left\{F_{t}\right\}_{t \in R_{+}}$, and $\sigma_{i}^{2}(i=1,2,3,4,5)$ represents the intensity of noise. Let $\varphi(\theta)$ be a continuous function on $[-\tau, 0]$, and let the 
solution $x(t)$ of system (2) satisfy the initial condition

$$
x(\theta)=\varphi(\theta)>0, \quad \theta \in[-\tau, 0] .
$$

Remark 1.1 (see [7]) In model (2), since $c_{0}(t)$ and $c_{e}(t)$ denote the concentrations of toxicant, we must have $0 \leq c_{0}(t) \leq 1$ and $0 \leq c_{e}(t) \leq 1$ for all $t \geq 0$. To this end, we need the following constraints: $k \leq g+m$ and $b \leq 1-e^{-h \gamma}$.

\section{Preliminaries}

Let $R_{+}=\{a \in R: a>0\}$, and let $C\left(\Omega \times[0,+\infty) ; R_{+}\right)$denote the family of continuous functions from $\Omega \times[0,+\infty)$ to $R_{+}$. In addition, for convenience, we also introduce some notations:

$$
\begin{aligned}
&\langle x(t)\rangle=t^{-1} \int_{0}^{t} x(s) d s, \quad x_{*}=\liminf _{t \rightarrow+\infty} x(t), \quad x^{*}=\limsup _{t \rightarrow+\infty} x(t), \\
& \eta= \frac{-1}{h \gamma \sqrt{\alpha}}\left(\arctan \left(\frac{\mu \sqrt{\alpha} e^{-k \gamma}}{1-e^{-k \gamma}}\right)-\arctan \left(\frac{\mu \sqrt{\alpha}}{1-e^{-k \gamma}}\right)\right), \\
& \xi= \frac{\mu^{2}\left(1-e^{-h \gamma}\right)^{3}\left(1+e^{-h \gamma}\right)}{2 h \gamma\left(\left(1-e^{-h \gamma}\right)^{2}+\alpha \mu^{2} e^{-2 h \gamma}\right)\left(\left(1-e^{-h \gamma}\right)^{2}+\alpha \mu^{2}\right)}, \\
& \nu=\left(\frac{k \mu}{h-g-m-b}\right)^{2}\left(\frac{1+e^{-(g+m+b) \gamma}}{2(g+m+b) \gamma\left(1-e^{-(g+m+b) \gamma}\right)}+\frac{1+e^{-h \gamma}}{2 h \gamma\left(1-e^{-h \gamma}\right)}\right. \\
&\left.\quad-\frac{2\left(1-e^{-(g+m+b+h) \gamma}\right)}{\left(1-e^{-h \gamma}\right)\left(1-e^{-(g+m+b) \gamma}\right)(g+m+b+h) \gamma}\right), \\
& A= r-\frac{r_{0} k \mu}{h(g+m+b) \gamma}-\lambda \eta-0.5 \sigma_{1}^{2}-0.5 \sigma_{2}^{2} \nu-0.5 \sigma_{5}^{2} \xi .
\end{aligned}
$$

\section{Definition 2.1}

(1) population $x(t)$ goes to extinction if $\lim _{t \rightarrow+\infty} x(t)=0$ a.s.;

(2) population $x(t)$ is nonpersistent in the mean if $\langle x(t)\rangle^{*}=0$;

(3) population $x(t)$ is weakly persistent if $x^{*}>0$;

(4) population $x(t)$ is strongly persistent in the mean if $\langle x(t)\rangle_{*}>0$.

Lemma 2.1 (see [25]) Consider the following subsystem of (2):

$$
\left\{\begin{array}{l}
d c_{0}(t)=\left(k c_{e}(t)-(g+m+b) c_{0}(t)\right) d t, \\
d c_{e}(t)=-h c_{e}(t) d t, \quad t \neq n \gamma, n \in Z^{+}, \\
\Delta c_{0}(t)=0, \quad \Delta c_{e}(t)=\mu, \quad t=n \gamma, n \in Z^{+} .
\end{array}\right.
$$

Model (3) has a unique positive $\gamma$-periodic solution $\left(\widetilde{c}_{0}(t), \widetilde{c}_{e}(t)\right)$, and for each solution $\left(c_{0}(t), c_{e}(t)\right)$ of model $(2), c_{0}(t) \rightarrow \widetilde{c}_{0}(t)$ and $c_{e}(t) \rightarrow \widetilde{c}_{e}(t)$ as $t \rightarrow+\infty$. Moreover, $c_{0}(t)>\widetilde{c}_{0}(t)$ and $c_{e}(t)>\widetilde{c}_{e}(t)$, where

$$
\left\{\begin{array}{l}
\widetilde{c_{0}}(t)=\widetilde{c_{0}}(0) e^{-(g+m+b)(t-n \gamma)}+\frac{k \mu\left(e^{-(g+m+b)(t-n \gamma)}-e^{-h(t-n \gamma)}\right)}{(h-g-m-b)\left(1-e^{-h \gamma}\right)}, \\
\widetilde{c_{e}}(t)=\frac{\mu e^{-h(t-n \gamma)}}{1-e^{-h \gamma}}, \\
\widetilde{c_{0}}(0)=\frac{k \mu\left(e^{-(g+m+b) \gamma}-e^{-h \gamma}\right)}{(h-g-m-b)\left(1-e^{-(g+m+b) \gamma}\right)\left(1-e^{-h \gamma}\right)}, \\
\widetilde{c_{e}}(0)=\frac{\mu}{1-e^{-h \gamma}}
\end{array}\right.
$$

for $t \in(n \gamma,(n+1) \gamma]$ and $n \in z^{+}$. 
Lemma 2.2 The positive $\gamma$-periodic solution $\left(\tilde{c}_{o}(t), \tilde{c}_{e}(t)\right)$ has the following properties:

$$
\begin{aligned}
& \lim _{t \rightarrow+\infty} t^{-1} \int_{0}^{t} \widetilde{c}_{0}(s) d s=\frac{k \mu}{h(g+m+b) \gamma}, \\
& \lim _{t \rightarrow+\infty} t^{-1} \int_{0}^{t} \widetilde{c}_{e}(s) d s=\frac{\mu}{h \gamma}, \quad \lim _{t \rightarrow+\infty} t^{-1} \int_{0}^{t} \frac{\widetilde{c}_{e}(s)}{1+\alpha \widetilde{c}_{e}^{2}(s)} d s=\eta, \\
& \lim _{t \rightarrow+\infty} t^{-1} \int_{0}^{t} \widetilde{c}_{0}^{2}(s) d s=\omega, \quad \lim _{t \rightarrow+\infty} t^{-1} \int_{0}^{t}\left(\frac{\widetilde{c}_{e}(s)}{1+\alpha \widetilde{c}_{e}^{2}(s)}\right)^{2} d s=\xi .
\end{aligned}
$$

Proof By the lemma of Yang [26] and Lemma 2.1 of Lan [9] we get

$$
\begin{aligned}
& \lim _{t \rightarrow+\infty} t^{-1} \int_{0}^{t} \widetilde{c}_{0}(s) d s=\frac{k \mu}{h(g+m+b) \gamma}, \\
& \lim _{t \rightarrow+\infty} t^{-1} \int_{0}^{t} \widetilde{c}_{e}(s) d s=\frac{\mu}{h \gamma}, \\
& \lim _{t \rightarrow+\infty} t^{-1} \int_{0}^{t} \frac{\widetilde{c}_{e}(s)}{1+\alpha \widetilde{c}_{e}^{2}(s)} d s=\eta .
\end{aligned}
$$

Next, we will prove the last two limits. From

$$
\begin{aligned}
& \tilde{c_{0}}(t)=\widetilde{c_{0}}(0) e^{-(g+m+b) t}+\frac{k \mu\left(e^{-(g+m+b) t}-e^{-h(t-n \gamma)}\right)}{(h-g-m-b)\left(1-e^{-h \gamma}\right)}, \\
& \tilde{c}_{e}(t)=\frac{\mu e^{-h t}}{1-e^{-h \gamma}}
\end{aligned}
$$

for all $t \in(0, \gamma]$ we have

$$
\begin{aligned}
\gamma^{-1} & \int_{0}^{\gamma} \tilde{c}_{0}^{2}(t) d t \\
= & \gamma^{-1} \int_{0}^{\gamma}\left(\tilde{c}_{0}(0) e^{-(g+m+b) t}+\frac{k \mu\left(e^{-(g+m+b) t}-e^{-h t}\right)}{(h-g-m-b)\left(1-e^{-h \gamma}\right)}\right)^{2} d t \\
= & \left(\frac{k \mu}{(h-g-m-b)\left(1-e^{-(g+m+b) \gamma}\right)}\right)^{2} \gamma^{-1} \int_{0}^{\gamma} e^{-2(g+m+b) t} d t \\
& +\left(\frac{k \mu}{(h-g-m-b)\left(1-e^{-h \gamma}\right)}\right)^{2} \gamma^{-1} \int_{0}^{\gamma} e^{-2 h \gamma} d t \\
& -2\left(\frac{(k \mu)^{2}}{(h-g-m-b)^{2}\left(1-e^{-(g+m+b) \gamma}\right)\left(1-e^{-h \gamma}\right)}\right) \gamma^{-1} \int_{0}^{\gamma} e^{-(g+m+b+h) t} d t \\
= & \left(\frac{k \mu}{(h-g-m-b)\left(1-e^{-(g+m+b) \gamma}\right)}\right)^{2} \frac{1-e^{-2(g+m+b) \gamma}}{2(g+m+b) \gamma} \\
& -\left(\frac{k \mu}{(h-g-m-b)\left(1-e^{-h \gamma}\right)}\right)^{2} \frac{1-e^{-2 h \gamma}}{2 h \gamma} \\
& -2\left(\frac{(k \mu)^{2}}{(h-g-m-b)^{2}\left(1-e^{-(g+m+b) \gamma}\right)\left(1-e^{-h \gamma}\right)}\right) \frac{1-e^{-(g+m+b+h) \gamma}}{(g+m+b+h) \gamma} \\
= & \nu .
\end{aligned}
$$


Similarly,

$$
\begin{aligned}
\gamma^{-1} \int_{0}^{\gamma}\left(\frac{\tilde{c}_{e}(s)}{1+\alpha \tilde{c}_{e}^{2}(s)}\right)^{2} d s & =\frac{-1}{h \gamma} \int_{0}^{\gamma} \frac{\tilde{c}_{e}(s)\left(-h \tilde{c}_{e}(s)\right)}{\left(1+\alpha \tilde{c}_{e}^{2}(s)\right)^{2}} d s \\
& =\frac{-1}{2 h \gamma \alpha} \int_{0}^{\gamma} \frac{1}{\left(1+\alpha{\tilde{c_{e}}}^{2}(s)\right)^{2}} d\left(1+\alpha{\tilde{c_{e}}}^{2}(s)\right) \\
& =\frac{1}{2 h \gamma \alpha}\left(\frac{1}{1+\alpha \tilde{c}_{e}^{2}(\gamma)}-\frac{1}{1+\alpha{\tilde{c_{e}}}^{2}(0)}\right) \\
& =\xi .
\end{aligned}
$$

In view of the periodicity of $\tilde{c}_{0}(t)$ and $\tilde{c}_{e}(t)$ we can observe that

$$
\begin{aligned}
& \lim _{t \rightarrow+\infty} t^{-1} \int_{0}^{t} \tilde{c}_{0}^{2}(s) d s=\gamma^{-1} \int_{0}^{\gamma} \tilde{c}_{0}^{2}(s) d s=v, \\
& \lim _{t \rightarrow+\infty} t^{-1} \int_{0}^{t}\left(\frac{\tilde{c}_{e}(s)}{1+\alpha \tilde{c}_{e}^{2}(s)}\right)^{2} d s=\gamma^{-1} \int_{0}^{\gamma}\left(\frac{\tilde{c}_{e}(s)}{1+\alpha \tilde{c}_{e}^{2}(s)}\right)^{2} d s=\xi .
\end{aligned}
$$

Lemma 2.3 (see [27]) Suppose that $x(t) \in C\left(\Omega \times[0,+\infty) ; R_{+}\right)$.

(1) If there are $\lambda$ and positive constants $\lambda_{0}, T$ such that

$$
\ln x(t) \leq \lambda t-\lambda_{0} \int_{0}^{t} x(s) d s+\sum_{i=1}^{n} \beta_{i} d B_{i}(t), \quad t \geq T
$$

where $\beta_{i}(1 \leq i \leq n)$ are constants, then

$$
\left\{\begin{array}{l}
\lim \sup _{t \rightarrow+\infty} t^{-1} \int_{0}^{t} x(s) d s \leq \frac{\lambda}{\lambda_{0}} \quad \text { a.s. }, \lambda \geq 0, \\
\lim _{t \rightarrow+\infty} x(t)=0 \quad \text { a.s. }, \lambda<0 .
\end{array}\right.
$$

(2) If there are positive constants $\lambda, \lambda_{0}$, and $T$ such that

$$
\ln x(t) \geq \lambda t-\lambda_{0} \int_{0}^{t} x(s) d s+\sum_{i=1}^{n} \beta_{i} d B_{i}(t), \quad t \geq T,
$$

then $\liminf _{t \rightarrow+\infty} t^{-1} \int_{0}^{t} x(s) d s \leq \frac{\lambda}{\lambda_{0}}$ a.s.

Lemma 2.4 (see[14]) Consider the stochastic differential equation

$$
d x(t)=x(t)(r-a x(t)) d t+\sigma x(t) d B(t)
$$

where $r, a$, and $\sigma$ are positive constants. If $r-0.5 \sigma^{2}>0$, then

$$
\lim _{t \rightarrow+\infty}\langle x(t)\rangle=\frac{r-0.5 \sigma^{2}}{a}, \quad \lim _{t \rightarrow+\infty} \frac{\ln x(t)}{t}=0 \quad \text { a.s. }
$$

\section{Main results}

Lemma 3.1 For any given initial value $x(\theta)=\varphi(\theta) \in C\left([-\tau, 0] ; R_{+}\right)$, the first equation of model (2) has a unique global positive solution $x(t)$. Moreover, $\lim _{\sup } \rightarrow+\infty \frac{\ln x(t)}{t} \leq 0$ a.s. 
Proof Considering the stochastic differential equation

$$
\begin{aligned}
d x(t)= & x(t)\left(r-r_{0} c_{0}(t)-a x(t)-c x(t-\tau)-\frac{\lambda c_{e}(t)}{1+\alpha c_{e}^{2}(t)}\right) d t \\
& +\sigma_{1} x(t) d B_{1}(t)+\sigma_{2} c_{0}(t) x(t) d B_{2}(t)+\sigma_{3} x^{2}(t) d B_{3}(t) \\
& +\sigma_{4} x(t) x(t-\tau) d B_{4}(t)+\frac{\sigma_{5} x(t) c_{e}(t)}{1+\alpha c_{e}^{2}(t)} d B_{5}(t) .
\end{aligned}
$$

The proof of the existence and uniqueness of the global positive solution of the equation is similar to that of Dai et al. [24] by defining the nonnegative function

$$
V_{1}(x)=\sqrt{x}-1-0.5 \ln x+\frac{1+\sigma_{4}^{2}}{4} \int_{t-\tau}^{t} x^{2}(s) d s, \quad x>0 .
$$

Hence we omit it.

Next, we will focus on proving that $\lim _{t \rightarrow+\infty} \frac{\ln x(t)}{t} \leq 0$ a.s. Applying Itồ's formula to $\ln x(t)$, we obtain

$$
\begin{aligned}
d \ln x(t)= & \left(r-r_{0} c_{0}(t)-a x(t)-c x(t-\tau)-\frac{\lambda c_{e}(t)}{1+\alpha c_{e}^{2}(t)}\right) d t \\
& -\frac{1}{2}\left(\sigma_{1}^{2}+\sigma_{2}^{2} C_{0}^{2}(t)+\sigma_{3}^{2} x^{2}(t)+\sigma_{4}^{2} x^{2}(t-\tau)+\sigma_{5}^{2}\left(\frac{c_{e}(t)}{1+\alpha c_{e}^{2}(t)}\right)^{2}\right) d t \\
& +\sigma_{1} d B_{1}(t)+\sigma_{2} c_{0}(t) d B_{2}(t)+\sigma_{3} x(t) d B_{3}(t) \\
& +\sigma_{4} x(t-\tau) d B_{4}(t)+\frac{\sigma_{5} c_{e}(t)}{1+\alpha c_{e}^{2}(t)} d B_{5}(t) .
\end{aligned}
$$

Using Itô’s formula again, we get

$$
\begin{aligned}
d e^{t} \ln x(t)= & e^{t} \ln x(t) d t+e^{t} d(\ln x(t)) \\
= & e^{t} \ln x(t)+e^{t}\left(r-r_{0} c_{0}(t)-a x(t)-c x(t-\tau)-\frac{\lambda c_{e}(t)}{1+\alpha c_{e}^{2}(t)}\right) d t \\
& -0.5 e^{t}\left(\sigma_{1}^{2}+\sigma_{2}^{2} C_{0}^{2}(t)+\sigma_{3}^{2} x^{2}(t)+\sigma_{4}^{2} x^{2}(t-\tau)+\sigma_{5}^{2}\left(\frac{c_{e}(t)}{1+\alpha c_{e}^{2}(t)}\right)^{2}\right) d t \\
& +e^{t}\left(\sigma_{1} d B_{1}(t)+\sigma_{2} c_{0}(t) d B_{2}(t)+\sigma_{3} x(t) d B_{3}(t)+\sigma_{4} x(t-\tau) d B_{4}(t)\right. \\
& \left.+\frac{\sigma_{5} c_{e}(t)}{1+\alpha c_{e}^{2}(t)} d B_{5}(t)\right) .
\end{aligned}
$$

Integrating both sides of equality (6) from 0 to $t$, we get

$$
\begin{aligned}
e^{t} \ln x(t)= & \int_{0}^{t} e^{s}\left(r-r_{0} c_{0}(s)-a x(s)-c x(s-\tau)-\frac{\lambda c_{e}(s)}{1+\alpha c_{e}^{2}(s)}\right) d s \\
& -\int_{0}^{t} \frac{e^{s}}{2}\left(\sigma_{1}^{2}+\sigma_{2}^{2} C_{0}^{2}(s)+\sigma_{3}^{2} x^{2}(s)+\sigma_{4}^{2} x^{2}(s-\tau)+\sigma_{5}^{2}\left(\frac{c_{e}(s)}{1+\alpha c_{e}^{2}(s)}\right)^{2}\right) d s \\
& +\ln x(0)+M_{1}(t)+M_{2}(t)+M_{3}(t)+M_{4}(t)+M_{5}(t),
\end{aligned}
$$


where

$$
\begin{aligned}
& M_{1}(t)=\int_{0}^{t} \sigma_{1} e^{s} d B_{1}(s), \quad M_{2}(t)=\int_{0}^{t} \sigma_{2} e^{s} c_{0}(s) d B_{2}(s), \\
& M_{3}(t)=\int_{0}^{t} \sigma_{3} e^{s} x(s) d B_{3}(s), \quad M_{4}(t)=\int_{0}^{t} \sigma_{4} e^{s} x(s-\tau) d B_{4}(s), \\
& M_{5}(t)=\int_{0}^{t} \frac{\sigma_{5} e^{s} c_{e}(s)}{1+\alpha c_{e}^{2}(s)} d B_{5}(s) .
\end{aligned}
$$

Note that $M_{i}(t)(i=1,2,3,4,5)$ is a local martingale. Therefore the quadratic variation of $M_{i}(t)$ is

$$
\begin{aligned}
& \left\langle M_{1}(t), M_{1}(t)\right\rangle=\int_{0}^{t} \sigma_{1}^{2} e^{2 s} d s, \quad\left\langle M_{2}(t), M_{2}(t)\right\rangle=\int_{0}^{t} \sigma_{2}^{2} e^{2 s} c_{0}^{2}(s) d s, \\
& \left\langle M_{3}(t), M_{3}(t)\right\rangle=\int_{0}^{t} \sigma_{3}^{2} e^{2 s} x^{2}(s) d s, \quad\left\langle M_{4}(t), M_{4}(t)\right\rangle=\int_{0}^{t} \sigma_{4}^{2} e^{2 s} x^{2}(s-\tau) d s, \\
& \left\langle M_{5}(t), M_{5}(t)\right\rangle=\int_{0}^{t} \sigma_{5}^{2} e^{2 s}\left(\frac{c_{e}(s)}{1+\alpha c_{e}^{2}(s)}\right)^{2} d s .
\end{aligned}
$$

Using the exponential martingale inequality, for all $\delta>0, \beta>0, T>0$, and $\theta>1$, we have

$$
P\left\{\sup _{0 \leq t \leq T}\left[M_{1}(t)-\frac{\delta}{2}\left\langle M_{1}(t), M_{1}(t)\right\rangle\right]>\beta\right\} \leq e^{-\alpha \beta} .
$$

Taking $\delta=e^{-k}, \beta=\theta e^{k} \ln k$, and $T=k$, we have

$$
P\left\{\sup _{0 \leq t \leq k}\left[M_{1}(t)-\frac{e^{-k}}{2}\left\langle M_{1}(t), M_{1}(t)\right\rangle\right]>\theta e^{k} \ln k\right\} \leq k^{-\theta} .
$$

By the Borel-Cantelli lemma there are an event $\Omega$ and positive integers $k_{1}=k_{1}(\omega)$ such that $P(\Omega)=1$ and for all $\omega \in \Omega$ and $k>k_{1}$, we have

$$
M_{1}(t) \leq \frac{e^{-k}}{2}\left\langle M_{1}(t), M_{1}(t)\right\rangle+\theta e^{k} \ln k, \quad 0 \leq t \leq k .
$$

Similarly,

$$
M_{i}(t) \leq \frac{e^{-k}}{2}\left\langle M_{i}(t), M_{i}(t)\right\rangle+\theta e^{k} \ln k, \quad 0 \leq t \leq k, i=2,3,4,5 .
$$

By (7) we get

$$
\begin{aligned}
e^{t} \ln x(t) \leq & \int_{0}^{t} e^{s}\left(r-r_{0} c_{0}(s)-a x(s)-c x(s-\tau)-\frac{\lambda c_{e}(s)}{1+\alpha c_{e}^{2}(s)}\right) d s \\
& -\int_{0}^{t} \frac{e^{s}}{2}\left(\sigma_{1}^{2}+\sigma_{2}^{2} C_{0}^{2}(s)+\sigma_{3}^{2} x^{2}(s)+\sigma_{4}^{2} x^{2}(s-\tau)+\sigma_{5}^{2}\left(\frac{c_{e}(s)}{1+\alpha c_{e}^{2}(s)}\right)^{2}\right) d s \\
& +\int_{0}^{t} \frac{e^{2 s-k}}{2}\left(\sigma_{1}^{2}+\sigma_{2}^{2} C_{0}^{2}(s)+\sigma_{3}^{2} x^{2}(s)+\sigma_{4}^{2} x^{2}(s-\tau)+\sigma_{5}^{2}\left(\frac{c_{e}(s)}{1+\alpha c_{e}^{2}(s)}\right)^{2}\right) d s \\
& +\ln x(0)+5 \theta e^{k} \ln k, \quad 0 \leq t \leq k .
\end{aligned}
$$


Because $a>0$, we can easily obtain that there is a positive constant $K>0$ such that $\ln x+$ $r-a x \leq K$, and from (8) it follows that

$$
e^{t} \ln x(t) \leq \ln x(0)+K\left(e^{t}-1\right)+5 \theta e^{k} \ln k, \quad 0 \leq t \leq k .
$$

For $k-1 \leq t \leq k$,

$$
\frac{\ln x(t)}{t} \leq \frac{\ln x(0)}{t e^{t}}+\frac{K\left(e^{t}-1\right)}{t e^{t}}+\frac{5 \theta e^{t} \ln k}{(k-1) e^{k-1}}
$$

and as $t \rightarrow+\infty(k \rightarrow+\infty)$, we have $\lim \sup _{t \rightarrow+\infty} \frac{\ln x(t)}{t} \leq 0$ a.s.

Theorem 3.2 Let $x(t)$ be the solution of system (2).

(i) If $A<0$, then the population $x(t)$ will die out almost surely.

(ii) If $A=0$, then the population $x(t)$ is nonpersistent in the mean almost surely.

(iii) If $A>0$, then the population $x(t)$ is weakly persistent almost surely.

Proof Integrating from 0 to $t$ both sides of equation (5), we get

$$
\begin{aligned}
\ln x(t) / x(0)= & \int_{0}^{t}\left(r-r_{0} c_{0}(s)-a x(s)-c x(s-\tau)-\frac{\lambda c_{e}(s)}{1+\alpha c_{e}^{2}(s)}\right) d s \\
& -\int_{0}^{t} \frac{1}{2}\left(\sigma_{1}^{2}+\sigma_{2}^{2} C_{0}^{2}(s)+\sigma_{3}^{2} x^{2}(s)+\sigma_{4}^{2} x^{2}(s-\tau)+\sigma_{5}^{2}\left(\frac{c_{e}(s)}{1+\alpha c_{e}^{2}(s)}\right)^{2}\right) d t \\
& +N_{1}(t)+N_{2}(t)+N_{3}(t)+N_{4}(t)+N_{5}(t),
\end{aligned}
$$

where

$$
\begin{aligned}
& N_{1}(t)=\int_{0}^{t} \sigma_{1} d B_{1}(s), \quad N_{2}(t)=\int_{0}^{t} \sigma_{2} c_{0}(s) d B_{2}(s), \\
& N_{3}(t)=\int_{0}^{t} \sigma_{3} x(s) d B_{3}(s), \quad N_{4}(t)=\int_{0}^{t} \sigma_{4} x(s-\tau) d B_{4}(s), \\
& N_{5}(t)=\int_{0}^{t} \frac{\sigma_{5} c_{e}(s)}{1+\alpha c_{e}^{2}(s)} d B_{5}(s) .
\end{aligned}
$$

The quadratic variations of $N_{2}(t), N_{3}(t), N_{4}(t)$, and $N_{5}(t)$ are

$$
\begin{array}{ll}
\left\langle N_{2}(t), N_{2}(t)\right\rangle=\int_{0}^{t} \sigma_{2}^{2} c_{0}^{2}(s) d s, \quad\left\langle N_{3}(t), N_{3}(t)\right\rangle=\int_{0}^{t} \sigma_{3}^{2} x^{2}(s) d s, \\
\left\langle N_{4}(t), N_{4}(t)\right\rangle=\int_{0}^{t} \sigma_{4}^{2} x^{2}(s-\tau) d s, & \left\langle N_{5}(t), N_{5}(t)\right\rangle=\int_{0}^{t}\left(\frac{\sigma_{5} c_{e}(s)}{1+\alpha c_{e}^{2}(s)}\right)^{2} d s .
\end{array}
$$

For all $t \in(n \gamma,(n+1) \gamma], n \in N_{+}$, we have

$$
\frac{1}{(n+1) \gamma} \int_{0}^{n \gamma} c_{0}^{2}(s) d s \leq \frac{1}{t} \int_{0}^{t} c_{0}^{2}(s) d s \leq \frac{1}{n \gamma} \int_{0}^{(n+1) \gamma} c_{0}^{2}(s) d s .
$$


Noting that $0 \leq c_{0}(t) \leq 1$ and $0 \leq c_{e}(t) \leq 1$, by Lemmas 2.1 and 2.2 we get

$$
\begin{aligned}
\lim _{t \rightarrow+\infty} \frac{\left\langle N_{2}(t), N_{2}(t)\right\rangle}{t} & =\lim _{t \rightarrow+\infty} \sigma_{2}^{2} t^{-1} \int_{0}^{t} c_{0}^{2}(s) d s \\
& =\sigma_{2}^{2} \gamma^{-1} \int_{0}^{\gamma} c_{0}^{2}(s) d s=v \sigma_{2}^{2}<+\infty .
\end{aligned}
$$

Similarly,

$$
\lim _{t \rightarrow+\infty} \frac{\left\langle N_{5}(t), N_{5}(t)\right\rangle}{t}=\sigma_{5}^{2} \gamma^{-1} \int_{0}^{\gamma}\left(\frac{c_{e}(s)}{1+\alpha c_{e}^{2}(s)}\right)^{2} d s=\sigma_{5}^{2} \xi<+\infty .
$$

By the strong law of large numbers it follows that

$$
\lim _{t \rightarrow+\infty} \frac{N_{1}(t)}{t}=0, \quad \lim _{t \rightarrow+\infty} \frac{N_{2}(t)}{t}=0, \quad \lim _{t \rightarrow+\infty} \frac{N_{5}(t)}{t}=0 \quad \text { a.s. }
$$

By the exponential martingale inequality, choosing $\delta=1, \beta=2 \ln k$, and $T=k$, have

$$
P\left\{\sup _{0 \leq t \leq k}\left[N_{i}(t)-\frac{1}{2}\left\langle N_{i}(t), N_{i}(t)\right\rangle\right]>2 \ln k\right\} \leq k^{-2}, \quad i=3,4 .
$$

It follows from Borel-Cantelli lemma that there exists a positive constant $k_{1}$ such that, for $k>k_{1}$, we have

$$
N_{i}(t) \leq 0.5\left\langle N_{i}(t), N_{i}(t)\right\rangle+2 \ln k, \quad i=3,4,0 \leq t \leq k
$$

Substituting this inequality into (9), we obtain that

$$
\begin{aligned}
\frac{\ln x(t) / x(0)}{t} \leq & r-0.5 \sigma_{1}^{2}-r_{0}\left\langle c_{0}(t)\right\rangle-a\langle x(t)\rangle-c|x(t-\tau)\rangle \\
& -\lambda\left\langle\frac{c_{e}(t)}{1+\alpha c_{e}^{2}(t)}\right\rangle-0.5 \sigma_{2}^{2}\left\langle c_{0}^{2}(t)\right\rangle-0.5 \sigma_{5}^{2}\left\langle\left(\frac{c_{e}(t)}{1+\alpha c_{e}^{2}(t)}\right)^{2}\right\rangle \\
& +\frac{N_{1}(t)+N_{2}(t)+N_{5}(t)}{t}+\frac{4 \ln k}{t} .
\end{aligned}
$$

For all $t \in(n \gamma,(n+1) \gamma]$, we have

$$
\begin{aligned}
& \frac{1}{(n+1) \gamma} \int_{0}^{n \gamma} c_{0}(s) d s \leq \frac{1}{t} \int_{0}^{t} c_{0}(s) d s \leq \frac{1}{n \gamma} \int_{0}^{(n+1) \gamma} c_{0}(s) d s, \\
& \frac{1}{(n+1) \gamma} \int_{0}^{n \gamma} \frac{c_{e}(s)}{1+\alpha c_{e}(s)} d s \leq \frac{1}{t} \int_{0}^{t} \frac{c_{e}(s)}{1+\alpha c_{e}(s)} d s \leq \frac{1}{n \gamma} \int_{0}^{(n+1) \gamma} \frac{c_{e}(s)}{1+\alpha c_{e}(s)} d s .
\end{aligned}
$$

It follows from Lemmas 2.1 and 2.2 that

$$
\begin{aligned}
& \lim _{t \rightarrow+\infty} t^{-1} \int_{0}^{t} c_{0}(s) d s=\gamma^{-1} \int_{0}^{\gamma} \tilde{c}_{0}(s) d s=\frac{k u}{h(g+m+b) \gamma}, \\
& \lim _{t \rightarrow+\infty} t^{-1} \int_{0}^{t} \frac{c_{e}(s)}{1+\alpha c_{e}^{2}(s)} d s=\gamma^{-1} \int_{0}^{\gamma} \frac{\tilde{c}_{e}(s)}{1+\alpha \tilde{c}_{e}^{2}(s)} d s=\eta .
\end{aligned}
$$


Let $k-1 \leq t \leq k, k \in N_{+}, A=r-0.5 \sigma_{1}^{2}-\frac{r_{0} k u}{h(g+m+b) \gamma}-\lambda \eta-0.5 \sigma_{2}^{2} v-0.5 \sigma_{5}^{2} \xi$. By virtue of (10), (11), (12), (14), and (15), for all $\epsilon>0$, there exists $T>0$ such that for all $t>T$ (i.e., $k>T)$, we have

$$
\begin{gathered}
-\frac{\epsilon}{4} \leq \frac{\ln x(0)}{t} \leq \frac{\epsilon}{4}, \quad-\frac{\epsilon}{4} \leq \frac{2 \ln k}{t} \leq \frac{\epsilon}{4}, \quad-\frac{\epsilon}{4} \leq \frac{N_{1}(t)+N_{2}(t)+N_{5}(t)}{t} \leq \frac{\epsilon}{4}, \\
A-\frac{\epsilon}{4} \leq r-\frac{\sigma_{1}^{2}}{2}-r_{0}\left\langle c_{0}(t)\right\rangle-\lambda\left\langle\frac{c_{e}(t)}{1+\alpha c_{e}^{2}(t)}\right\rangle-\frac{\sigma_{2}^{2}}{2}\left\langle c_{0}^{2}(t)\right\rangle \\
-\frac{\sigma_{5}^{2}}{2}\left\langle\left(\frac{c_{e}(t)}{1+\alpha c_{e}^{2}(t)}\right)^{2}\right\rangle \leq A+\frac{\epsilon}{4} .
\end{gathered}
$$

(i) If $A<0$, then for $\epsilon$ small enough such that $A+\epsilon<0$, by (13) and (16) we get

$$
\frac{\ln x(t)}{t} \leq A+\epsilon-a\langle x(t)\rangle+\frac{B_{1}(t)}{t}, \quad t \geq T
$$

Then by Lemma 2.3 we get $\lim _{t \rightarrow+\infty} x(t)=0$ a.s.

(ii) If $A=0$, then by (17) and Lemma 2.3 we have $\langle x(t)\rangle^{*} \leq \frac{\epsilon}{a}$ a.s. By the arbitrariness of $\epsilon$ we have $\langle x(t)\rangle^{*}=0$ a.s.

(iii) If $A>0$, then suppose the conclusion is not true, that is, $P\left(\left\{\omega \mid x^{*}(t, \omega)=0\right\}\right)>0$. Then for all $\omega \in\left\{\omega \mid x^{*}(t, \omega)=0\right\}$, we have $\lim _{t \rightarrow+\infty} x(t, \omega)=0$ a.s.

Because

$$
t^{-1} \int_{0}^{t} x(s-\tau) d s=t^{-1}\left[\int_{-\tau}^{0} x(s) d s+\int_{t}^{t-\tau} x(s) d s\right]+t^{-1} \int_{0}^{t} x(s) d s,
$$

it follows from $\lim _{t \rightarrow+\infty} x(\omega, t)=0$ a.s. that

$$
\begin{aligned}
& \lim _{t \rightarrow+\infty} t^{-1}\left[\int_{-\tau}^{0} x(s) d s+\int_{t}^{t-\tau} x(s) d s\right]=0 \quad \text { a.s., } \\
& \lim _{t \rightarrow+\infty}\langle x(t)\rangle=\lim _{t \rightarrow+\infty}\left\langle x^{2}(t)\right\rangle=\lim _{t \rightarrow+\infty}\langle x(t-\tau)\rangle=\lim _{t \rightarrow+\infty}\left\langle x^{2}(t-\tau)\right\rangle=0 .
\end{aligned}
$$

It follows from (9) that

$$
\begin{aligned}
\frac{\ln x(t)}{t}= & \frac{\ln x(0)}{t}+r-0.5 \sigma_{1}^{2}-r_{0}\left\langle c_{0}(t)\right\rangle-\lambda\left\langle\frac{c_{e}(t)}{1+\alpha c_{e}^{2}(t)}\right\rangle-0.5 \sigma_{2}^{2}\left\langle c_{0}^{2}(t)\right\rangle \\
& -0.5 \sigma_{5}^{2}\left\langle\left(\frac{c_{e}(t)}{1+\alpha c_{e}^{2}(t)}\right)^{2}\right\rangle-a\langle x(t)\rangle-c\langle x(t-\tau)\rangle-0.5 \sigma_{3}^{2}\left\langle x^{2}(t)\right\rangle \\
& -0.5\left\langle x^{2}(t-\tau)\right\rangle+\frac{N_{1}(t)+N_{2}(t)+N_{3}(t)+N_{4}(t)+N_{5}(t)}{t} .
\end{aligned}
$$

For all $\omega \in\left\{\omega \mid x^{*}(t, \omega)=0\right\}$, by (18) we get that $\lim _{t \rightarrow+\infty} \frac{N_{i}(t)}{t}=0$ a.s. $(i=1,2,3,4,5)$. Taking the limit superior of both sides of (19), we obtain that

$$
\limsup _{t \rightarrow+\infty} \frac{\ln x(t)}{t}=A>0 \quad \text { a.s., }
$$

which is a contradiction to the result of Lemma 3.1. 
Theorem 3.3 Suppose that $\sigma_{3}=\sigma_{4}=0$, and let $x(t)$ be the positive solution of model (2) with initial value $x(\theta)=\varphi(\theta) \in C\left([-\tau, 0], R_{+}\right)$. Then:

(I) If $A<0$, then the population $x$ goes to die out a.s., that is, $\lim _{t \rightarrow+\infty} x(t)=0$ a.s.

(II) If $A>0$, then the population $x$ is strongly persistent in the mean a.s.; moreover, $\lim _{t \rightarrow+\infty} t^{-1} \int_{0}^{t} x(s) d s=\frac{A}{a+c}$ a.s.

Proof If $\sigma_{3}=\sigma_{4}=0$, then by (19) we have

$$
\begin{aligned}
\frac{\ln x(t)}{t}= & \frac{\ln x(0)}{t}+r-0.5 \sigma_{1}^{2}-r_{0}\left\langle c_{0}(t)\right\rangle-\left\langle\frac{\lambda c_{e}(t)}{1+\alpha c_{e}^{2}(t)}\right\rangle-0.5 \sigma_{2}^{2}\left\langle c_{0}^{2}(t)\right\rangle \\
& -0.5 \sigma_{5}^{2}\left\langle\left(\frac{c_{e}(t)}{1+\alpha c_{e}^{2}(t)}\right)^{2}\right\rangle-a\langle x(t)\rangle-c\langle x(t-\tau)\rangle+\frac{N_{1}(t)+N_{2}(t)+N_{5}(t)}{t} .
\end{aligned}
$$

It follows from (12) and (16) that for all $\epsilon>0$, there exists a positive constant $T_{1}$ such that for $t>T_{1}$, we have

$$
\begin{aligned}
& \frac{\ln x(t)}{t} \leq A+\epsilon-a|x(t)\rangle-c|x(t-\tau)\rangle+\frac{B_{1}(t)}{t}, \\
& \frac{\ln x(t)}{t} \geq A-\epsilon-a\langle x(t)\rangle-c|x(t-\tau)\rangle+\frac{B_{1}(t)}{t} .
\end{aligned}
$$

(I) If $A<0$, then by Lemma 2.3 and (21) we have $\lim _{t \rightarrow+\infty} x(t)=0$ a.s.

Now we will prove (II). Let us consider the following auxiliary equation:

$$
\begin{aligned}
d y(t)= & y(t)\left(r-r_{0} c_{0}(t)-a y(t)-\frac{\lambda c_{e}(t)}{1+\alpha c_{e}^{2}(t)}\right) d t+\sigma_{1} y(t) d B_{1}(t) \\
& +\sigma_{2} c_{0}(t) y(t) d B_{2}(t)+\frac{\sigma_{5} y(t) c_{e}(t)}{1+\alpha c_{e}^{2}(t)} d B_{5}(t)
\end{aligned}
$$

where

$$
y(\theta)=\varphi(\theta)>0, \quad \theta \in[-\tau, 0] .
$$

It follows from the stochastic comparison theorem [14] that $x(t) \leq y(t)$. Hence, for all $\epsilon>0$, there is a positive constant $T_{2}$ such that for all $t>T_{2}$, we have

$$
\begin{aligned}
& \frac{\ln y(t)}{t} \leq A+\epsilon-a|y(t)\rangle+\frac{B_{1}(t)}{t}, \\
& \frac{\ln y(t)}{t} \geq A-\epsilon-a|y(t)\rangle+\frac{B_{1}(t)}{t} .
\end{aligned}
$$

By (24), (25), and Lemma 2.3 this implies that

$$
\frac{A-\epsilon}{a} \leq\left\langle\left. y(t)\right|_{*} \leq\left\langle\left. y(t)\right|^{*} \leq \frac{A+\epsilon}{a} .\right.\right.
$$

Due to the arbitrariness of $\epsilon$, from (26) we get

$$
\lim _{t \rightarrow+\infty} t^{-1} \int_{0}^{t} y(s) d s=\frac{A}{a} \quad \text { a.s. }
$$


Consequently,

$$
\lim _{t \rightarrow+\infty} t^{-1}\left[\int_{-\tau}^{0} y(s) d s+\int_{0}^{t-\tau} y(s) d s-\int_{0}^{t} y(s) d s\right]=0 \quad \text { a.s. }
$$

Since $x(t) \leq y(t)$, by (27) we easily see that

$$
\lim _{t \rightarrow+\infty} t^{-1}\left[\int_{-\tau}^{0} x(s) d s+\int_{0}^{t-\tau} x(s) d s-\int_{0}^{t} x(s) d s\right]=0 \quad \text { a.s. }
$$

It follows from (28) that, for all $\epsilon>0$, there exists a positive constant $T_{3}$ such that for $t>T_{3}$, have

$$
-\epsilon \leq t^{-1}\left[\int_{-\tau}^{0} x(s) d s+\int_{0}^{t-\tau} x(s) d s-\int_{0}^{t} x(s) d s\right] \leq \epsilon .
$$

For all $\epsilon>0$, there exists $T=\max \left\{T_{1}, T_{3}\right\}$ such that, for $t>T$, have

$$
\begin{aligned}
& \frac{\ln x(t)}{t} \leq A+2 \epsilon-(a+c)\langle x(t)\rangle+\frac{B_{1}(t)}{t}, \\
& \frac{\ln x(t)}{t} \geq A-2 \epsilon-(a+c)\langle x(t)\rangle+\frac{B_{1}(t)}{t} .
\end{aligned}
$$

By (29) and (30) we derive that

$$
\lim _{t \rightarrow+\infty} t^{-1} \int_{0}^{t} x(s) d s=\frac{A}{a+c} \quad \text { a.s. }
$$

Remark 3.4 From Theorems 3.2 and 3.3 we easily see that $A$ is the threshold of system (2) for the single-species population extinction and weak persistence. If $A<0$, then the population will be extinct, and if $A>0$, then the population is weakly persistent. Particularly, when $\sigma_{3}=\sigma_{4}=0, A$ is also the threshold of system (2) for the single-species population extinction and strong persistence; moreover, if $A>0$, then the single-species population is stable in the mean.

Remark 3.5 Note that from the expression for $A=r-\frac{r_{0} k \mu}{h(g+m+b) \gamma}-\lambda \eta-0.5 \sigma_{1}^{2}-0.5 \sigma_{2}^{2} v-$ $0.5 \sigma_{5}^{2} \xi$ we can find that the parameters $\mu$ and $\gamma$ obviously affect the persistence and extinction of system (2), that is, we can control the persistence and extinction of population $\mathrm{x}$ by controlling the toxicant input amount $\mu$ and the period of pulse input toxicant $\gamma$.

Remark 3.6 Theorems 3.2 and 3.3 show that the persistence population $\mathrm{x}$ of deterministic system (1) may be extinct when $\sigma_{1}, \sigma_{2}$, and $\sigma_{5}$ are large enough; however, $\tau, \sigma_{3}$, and $\sigma_{4}$ have no effect on the persistence and extinction for population $x$.

\section{Numerical simulations and discussion}

Next, we show the numerical simulation results to illustrate the accuracy of analytical results in the previous section by using the famous Milstein method [28]. We choose the parameters of system (2) as follows:

$$
r=0.4, \quad r_{0}=0.3, \quad a=0.4, \quad c=0.4, \quad \lambda=0.8, \quad k=0.6,
$$




$$
g=0.3, \quad m=0.3, \quad b=0.1, \quad h=0.4, \quad \alpha=0.4, \quad \tau=1 .
$$

To understand the effects of white noise and pulse toxicant input on population dynamics, we change the values of $\mu, \gamma$, and $\sigma_{i}(i=1,2,3,4,5)$.

(1) We firstly adopt $u=0.3, \sigma_{1}=0.3, \sigma_{2}=0.2, \sigma_{3}=0.2, \sigma_{4}=0.4, \sigma_{5}=0.4$. If $\gamma=5$, then simple calculation shows that $A=0.1955>0$. In view of the Theorem 3.2, we obtain that the single-species population $x$ is weakly persistent; see Fig. 1 . If $\gamma=2$, then by computing we have $A=-0.0439<0$, so condition (i) of Theorem 3.2 holds, that is, the population $x$ of system (2) will extinct (see Fig. 2).

(2) Next, to analyze the effect of the toxicant input amount each time $\mu$ on the persistence of the single species, we adopt $u=0.8, \sigma_{1}=0.3, \sigma_{2}=0.2, \sigma_{3}=0.2, \sigma_{4}=0.4, \sigma_{5}=0.4$, and $\gamma=5$. Simple calculation shows that $A=-0.0555<0$, and from Theorem 3.2 it follows that the population $x$ will die out a.s. (see Fig. 3). From Figs. 1 and 3 we can see that the population $x$ will die out when the environmental toxicant amount of each time $\mu$ is large enough.

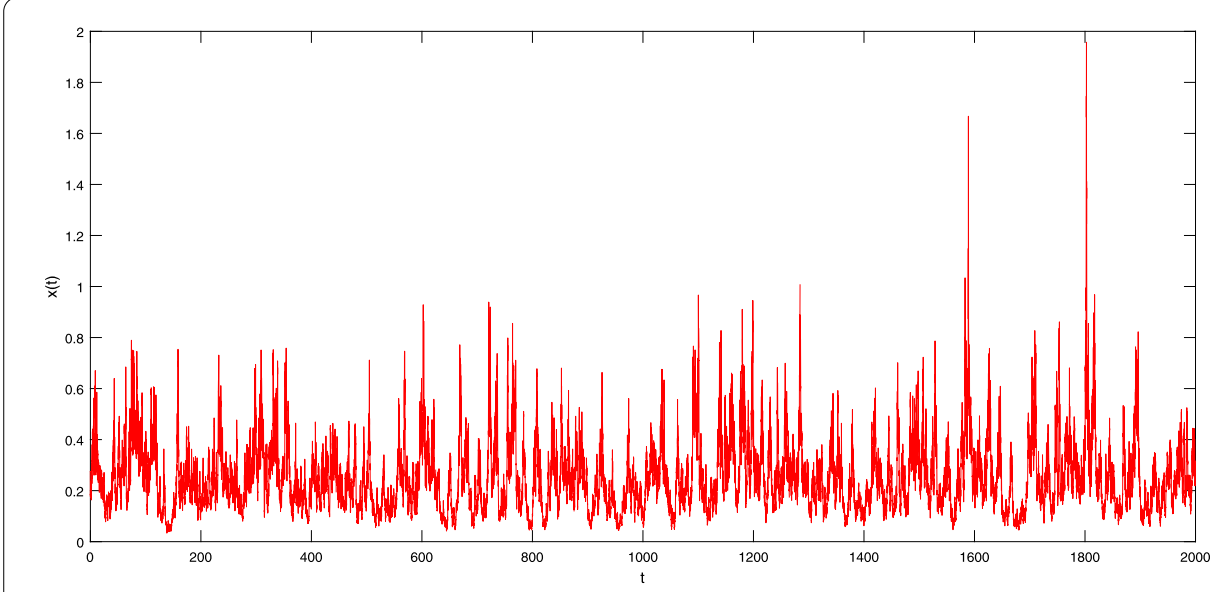

Figure 1 Solution of system (2) with $\sigma_{1}=0.3, \sigma_{2}=0.2, \sigma_{3}=0.2, \sigma_{4}=0.4, \sigma_{5}=0.4, u=0.3, \gamma=5$

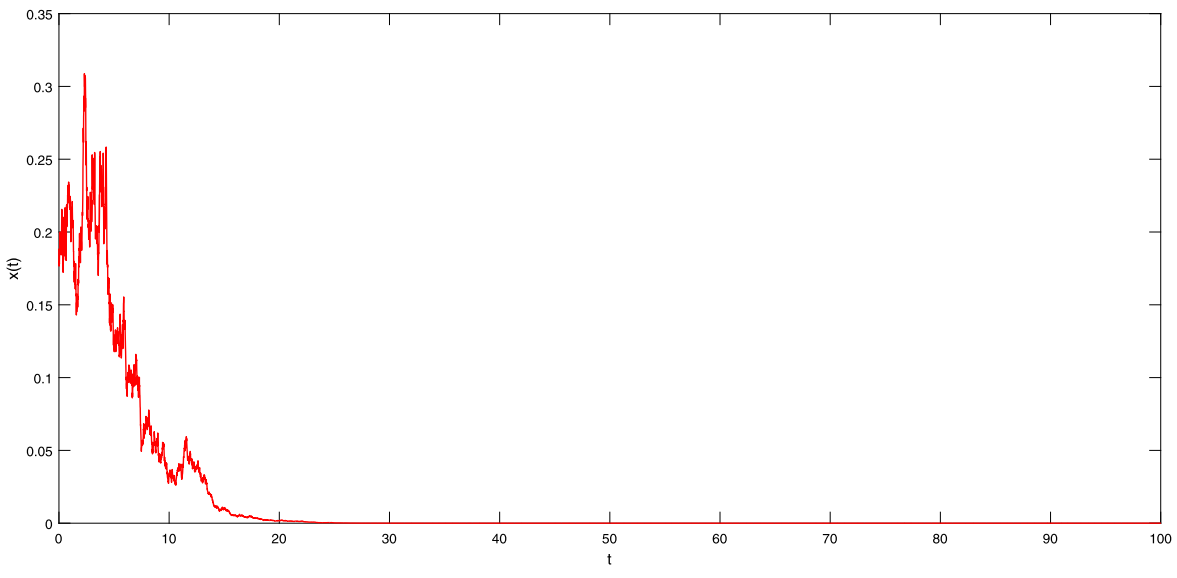

Figure 2 Solution of system (2) with $\sigma_{1}=0.3, \sigma_{2}=0.2, \sigma_{3}=0.2, \sigma_{4}=0.4, \sigma_{5}=0.4, u=0.3, \gamma=2$ 


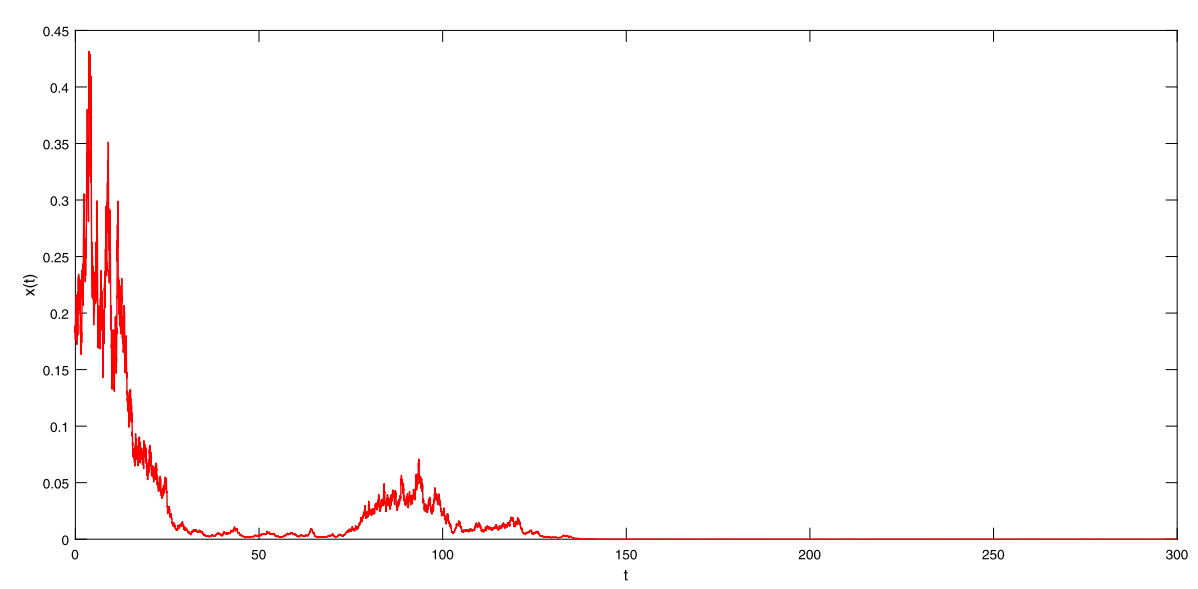

Figure 3 Solution of system (2) with $\sigma_{1}=0.3, \sigma_{2}=0.2, \sigma_{3}=0.2, \sigma_{4}=0.4, \sigma_{5}=0.4, u=0.8, \gamma=5$

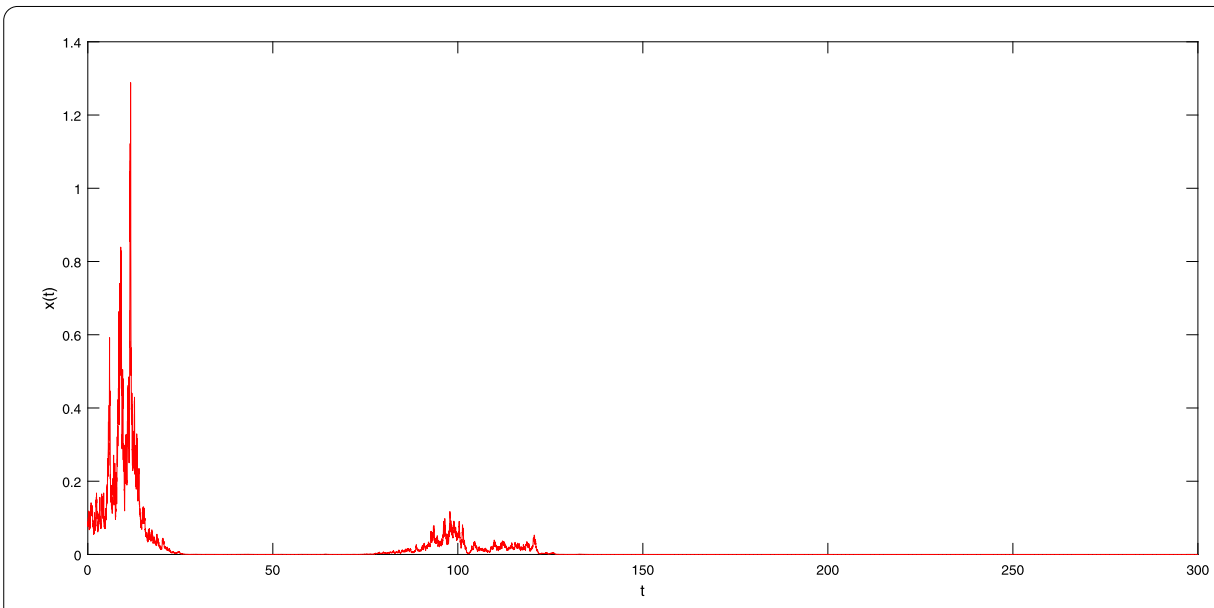

Figure 4 Solution of system (2) with $\sigma_{1}=0.8, \sigma_{2}=0.2, \sigma_{3}=0.2, \sigma_{4}=0.6, \sigma_{5}=0.6, u=0.3, \gamma=5$

(3) On the other hand, we will focus on the influence of the intensity of white noises on the survival for the population $x$. We adopt $u=0.3$ and $\gamma=5$ and suppose that $\sigma_{1}=0.8$, $\sigma_{2}=0.2, \sigma_{3}=0.2, \sigma_{4}=0.6$, and $\sigma_{5}=0.6$. Simple calculation shows that $A=-0.0823<0$, so that the population $x$ will die out (see Fig. 4). Suppose $\sigma_{1}=0.3, \sigma_{2}=0.2, \sigma_{3}=0, \sigma_{4}=0$, and $\sigma_{5}=0.4$. Then $A=0.1955>0$, and by Theorem 3.3 we obtain that the population $x$ is strongly persistent in the mean; moreover, $\lim _{t \rightarrow+\infty} t^{-1} \int_{0}^{t} x(s) d s=0.2443$, that is, the population is stable in the mean (see Fig. 5). From Figs. 1 and 5 we can see that the population $x$ will die out when $\sigma_{1}, \sigma_{2}, \sigma_{5}$ are large enough, but $\sigma_{3}$ and $\sigma_{4}$ have no effect on the survival of the population $x$.

\section{Conclusions}

We studied a stochastic impulsive single-species population model with psychological effect and delay in pollution environment. We obtained the threshold $A$ between weak persistence and extinction. Particularly, $A$ is also a threshold of system (2) for the strong persistence and extinction when $\sigma_{3}=\sigma_{4}=0$. These results have revealed that the environmental noise, the impulsive period, and the amount of toxicant input for each time have 


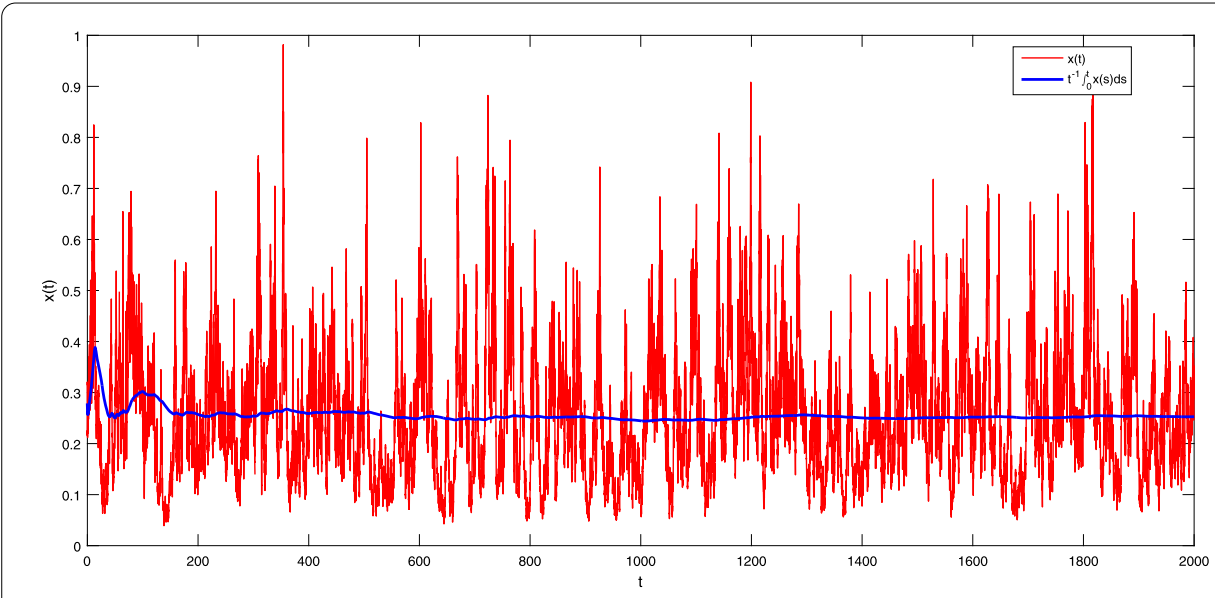

Figure 5 Solution of system (2) with $\sigma_{1}=0.3, \sigma_{2}=0.2, \sigma_{3}=0, \sigma_{4}=0, \sigma_{5}=0.4, u=0.3, \gamma=5$

an influence on the persistence and extinction for the single-species population and show that the delay $\tau$ and the intensities of the white noises $\sigma_{3}^{2}$ and $\sigma_{4}^{2}$ have no effect on the persistence and extinction for the population $x$. Therefore Theorems 3.2 and 3.3 extend the corresponding results in $[8,9]$.

However, there are still many interesting questions to be further studied. On the one hand, we can propose more realistic and complex models, and the method used in this paper can be also applied to study other interesting models, such as Gompertz models, Gilpin-Ayala models, and so on. On the other hand, in recent years, optimal harvesting problems have received a lot of attention [29], and hence we could add harvest items to model (2) and discuss the optimal harvesting problem. We leave these investigations for future work.

\section{Acknowledgements}

The authors are very grateful to the Youth Science and technology talent growth project of Guizhou Provincial Department of Education (KY[2018]341, KY[2015]456) and the Project for Innovative Research Groups of Guizhou Province of China (KY[2016]051).

\section{Funding}

This paper is supported by the Youth Science and technology talent growth project of Guizhou Provincial Department of Education (KY[2018]341, KY[2017]318, KY[2015]456) and the Project for Innovative Research Groups of Guizhou Province of China (KY[2016]051).

\section{Availability of data and materials}

This paper focuses on theoretical analysis and does not involve data.

\section{Competing interests}

The authors declare that they have no competing interests.

\section{Authors' contributions}

All authors contributed equally to the writing of this paper. The authors read and approved the final manuscript.

\section{Author details}

${ }^{1}$ School of Data Science of TongRen University, 554300 Tongren, P.R. China. ${ }^{2}$ Tongren Preschool Education College, 554300 Tongren, P.R. China.

\section{Publisher's Note}

Springer Nature remains neutral with regard to jurisdictional claims in published maps and institutional affiliations. 


\section{References}

1. Hallam, T., Clark, R.C.: Effects of toxicants on populations: a qualitative approach I. Equilibrium environmental exposure. Ecol. Model. 8, 291-304 (1983)

2. Hallam, T., Clark, C., Jordan, G.: Effects of toxicants on populations: a qualitative approach II. First order kinetics, J. Math. Biol. 18, 25-37 (1983)

3. Hallam, T., Deluna, J.: Effects of toxicants on populations: a qualitative approach III. Environmental and food chain pathways. J. Theor. Biol. 109, 411-429 (1984)

4. Lika, K., Hallam, T.: Modeling the effects of toxicants on a fish population in a spatially heterogeneous environment: II. Lethal effects. Nonlinear Anal. 30, 1709-1719 (1997)

5. Xiao, Y., Chen, L.: Effects of toxicants on a stage-structured population growth model. Appl. Math. Comput. 123 63-73 (2001)

6. Gard, T.: A stochastic model for the effects of toxicants on populations. Ecol. Model. 51(3-4), 273-280 (1990)

7. Jiao, J.J., Chen, L.X.: The extinction threshold on a single population model with pulse input of environmental toxin in a polluted environment. Math. Appl. 22, 11-19 (2009)

8. Wei, F., Chen, L.: Psychological effect on single-species population models in a polluted environment. Math. Biosci. 290, 22-30 (2017)

9. Lan, G., Wei, C., Zhang, S.: Long time behaviors of single-species population models with psychological effect and impulsive toxicant in polluted environments. Physica A 521, 828-842 (2019)

10. Liu, M., Wang, K.: Persistence and extinction in stochastic non-autonomous logistic systems. J. Math. Anal. Appl. 375 , 443-457 (2011)

11. May, R.M.: Stability and Complexity in Model Ecosystems. Princeton University Press, Princeton (2001)

12. Liu, M., Wang, K.: Persistence, extinction and global asymptotical stability of a non-autonomous predator-prey model with random perturbation. Appl. Math. Model. 36, 5344-5353 (2012)

13. Liu, M., Wang, K., Liu, Y.: Long term behaviors of stochastic single-species growth models in a polluted environment II. Appl. Math. Model. 35, 752-762 (2011)

14. Liu, M., Wang, K., Wu, Q.: Survival analysis of stochastic competitive models in a polluted environment and stochastic competitive exclusion principle. Bull. Math. Biol. 73, 1969-2012 (2011)

15. Liu, M., Wang, K.: Persistence and extinction of a stochastic single-species population model in a polluted environment with impulsive toxicant input. Electron. J. Differ. Equ. 230, 823 (2013)

16. Liu, Q., Chen, Q.M.: Dynamics of stochastic delay Lotka-Volterra systems with impulsive toxicant input and Lévy noise in polluted environments. Appl. Math. Comput. 256, 52-67 (2015)

17. Liu, M., Du, C., Deng, M.: Persistence and extinction of a modified Leslie-Gower Holling-type II stochastic predator-prey model with impulsive toxicant input in polluted environments. Nonlinear Anal. Hybrid Syst. 27, 177-190 (2018)

18. Ji, C.Y., Jiang, D.Q., Shi, N.Z.: Analysis of a predator-prey model with modified Leslie-Gower and Holling-type II schemes with stochastic perturbation. J. Math. Anal. Appl. 359(2), 482-498 (2009)

19. Huang, Y., Liu, Q., Liu, Y.L.: Global asymptotic stability of a general stochastic Lotka-Volterra system with delays. Appl. Math. Lett. 26, 175-178 (2013)

20. Kuang, Y.: Delay Differential Equations with Applications in Population Dynamics. Academic Press, New York (1993)

21. Liu, M., Bai, C.: On a stochastic delayed predator-prey model with Lévy jumps. Appl. Math. Comput. 228, 563-570 (2014)

22. Li, X., Mao, X., Shen, Y.: Approximate solutions of stochastic differential delay equations with Markovian switching. J. Differ. Equ. Appl. 16, 195-207 (2010)

23. Liu, Y.L., Liu, Q., Liu, Z.H.: Dynamical behaviors of a stochastic delay logistic system with impulsive toxicant input in a polluted environment. J. Theor. Biol. 329, 1-5 (2013)

24. Dai, X.J., Mao, Z., Li, X.J.: A stochastic prey-predator model with time-dependent delays. Adv. Differ. Equ. 2017(1), 297 (2017)

25. Liu, B., Chen, L.S., Zhang, Y.Z.: The effects of impulsive toxicant input on a population in a polluted environment J. Biol. Syst. 11, 265-274 (2013)

26. Yang, X.F., Jin, Z., Xue, Y.K.: Weak average persistence and extinction of a predator-prey system in a polluted environment with impulsive toxicant input. Chaos Solitons Fractals 31, 726-735 (2007)

27. Liu, M., Bai, C.: Persistence and extinction of a stochastic cooperative model in a polluted environment with pulse toxicant input. Filomat 29, 1329-1342 (2015)

28. Higham, D.: An algorithmic introduction to numerical simulation of stochastic differential equations. SIAM Rev. 43, 525-546 (2001)

29. Zou, X.L., Li, W.X., Wang, K.: Ergodic method on optimal harvesting for a stochastic Gompertz-type diffusion process. Appl. Math. Lett. 26, 170-174 (2012) 